Dear Author,

Please, note that changes made to the HTML content will be added to the article before publication, but are not reflected in this PDF.

Note also that this file should not be used for submitting corrections. 


\title{
Surface-induced vacancy loops and damage dispersion in irradiated Fe thin films
}

\author{
M.J. Aliaga ${ }^{a}$, R. Schäublin ${ }^{\text {b }}$, J.F. Löffler ${ }^{b}$, M.J. Caturla ${ }^{a, *}$ \\ ${ }^{a}$ Dept. Física Aplicada, Facultad de Ciencias, Fase II, Universidad de Alicante, Alicante E-03690, Spain \\ ${ }^{\mathrm{b}}$ Laboratory of Metal Physics and Technology, Department of Materials, ETH Zurich, 8093 Zurich, Switzerland
}

\section{A R T I C L E I N F O}

\section{Article history:}

Received 20 May 2015

Revised 21 July 2015

Accepted 28 August 2015

Available online $\mathrm{xxxx}$

\section{Keywords:}

Molecular dynamics simulations

Ion irradiation

In situ transmission electron microscopy

Defects

Microstructure

\begin{abstract}
A B S T R A C T
Transmission electron microscopy (TEM) in situ ion implantation is a convenient way to study radiation damage, but it is biased by the proximity of the free surfaces of the electron transparent thin sample. In this work this bias was investigated by performing irradiation of Fe in thin foil and bulk form with ions of energies between $50 \mathrm{keV}$ and $100 \mathrm{keV}$ using molecular dynamics simulations. The damage resulting from the subsequent displacement cascades differs significantly between the two sample geometries. The most remarkable difference is in the resulting $\langle 100\rangle$ vacancy loops. Both their size and frequency are much greater in thin films, with loops reaching $4 \mathrm{~nm}$ in size. This is due to an imbalance between the number of vacancies and self-interstitials produced, since the faster self-interstitials can escape to the surfaces and remain there as ad-atoms. In addition, the self-interstitial clusters are smaller for thin foils and there is a larger dispersion of the induced damage in terms of defect number, defect clustering and defect morphology. The study discusses the impact of these results on the study of radiation effects during in situ experiments.
\end{abstract}

(c) 2015 Published by Elsevier Ltd. on behalf of Acta Materialia Inc.

\section{Introduction}

Ion implantation is a common way to produce defects in materials in a controlled manner, in terms of particle type and energy, irradiation dose, dose rate, and temperature. It is therefore a very valuable tool for investigating radiation effects in materials with a high degree of control of all involved variables [1]. The damage produced by implantation can be characterized using transmission electron microscopy (TEM), which provides information about the irradiation-induced defects, their number density, size, and possibly their type for those defects that are larger than about $1 \mathrm{~nm}$ [2]. In some facilities, such as JANNuS at CEA in France [3] or the IVEM-Tandem Facility at Argonne National Laboratory [4], it is possible to perform ion irradiation experiments in situ in a TEM, thus allowing observation while irradiating [5]. This technique is being used to validate simulation models of radiation effects to predict the damage produced by the $14 \mathrm{MeV}$ neutrons expected in future fusion reactors [1], with a particular focus on ferritic materials because of their good resistance to irradiation relative, for instance, to austenitic stainless steels.

\footnotetext{
* Corresponding author.

E-mail address: mj.caturla@ua.es (M.J. Caturla).
}

The difficulty of extrapolating the results of such in situ experiments to the effects of radiation in bulk materials was recognized long ago [6]. This difficulty arises because, to be able to use conventional TEM (CTEM) for the characterization of defects via diffraction contrast, the sample must be thin enough to minimize electron absorption and inelastic scattering that blur the image, but thick enough to reduce the effect of the free surfaces, implying a thickness of at least a few tens of nanometers but less than about $80 \mathrm{~nm}$ [7]. Free surfaces have indeed an impact on the elastic fields of defects because of the so-called image forces, which alter the configuration of the defects, and their interaction and migration. Surfaces affect the irradiation-induced microstructure also simply because defects produced in their vicinity can annihilate there if the defects migrate to the free surfaces. It is known experimentally that in the case of ferritic steels irradiation generates $1 / 2\langle 111\rangle$ and $\langle 100\rangle$ dislocation loops [8]. Because the activation energy for migration of $1 / 2\langle 111\rangle$ loops along $\langle 111\rangle$ directions is low, as shown both experimentally [9] and in computer simulations $[10,11]$, they migrate rapidly and can thus easily meet a surface where they will disappear. Note that if the foil normal is oriented close to a $\langle 111\rangle$ or $\langle 100\rangle$ direction all $1 / 2\langle 111\rangle$ loops can disappear at surfaces, while only half of them will escape if the orientation of the foil is $\langle 110\rangle$ [12]. The nature, vacancy or self-interstitial, of the loops observed after irradiation at low doses is controversial due to the difficulty of analyzing them when they are small. For 
larger sizes (beyond a few nanometers) obtained at higher doses, they have been identified as interstitial loops [13-16]. At low doses but with heavy ions of low energy, large $\langle 100\rangle$ vacancy loops located close to the surface have also been identified $[13,16]$.

The damage produced by energetic recoils in bulk bcc Fe has been extensively studied by different research groups [17-20] using molecular dynamics (MD) simulations; for a review, see Ref. [21]. There is a good apparent understanding of the formation of interstitial clusters. For instance, recent simulations by Calder et al. [22] have shown how large interstitial clusters are produced in the early stages of the collision cascade in bulk Fe due to the interaction between shock waves. Sand et al. [23] have also shown that a scaling law can be obtained for the size of self-interstitial clusters as a function of recoil energy in bulk tungsten. However, much less is known regarding vacancy clustering and the effect of surfaces in bcc metals, which is relevant to thin foil irradiation.

The effect of free surfaces in fcc metals has been studied by several groups. The work performed in the 1990s by Ghaly and Averback [24-26] revealed how the presence of the surface can change the morphology of the damage produced by a collision cascade with respect to the bulk. Later, Nordlund et al. [25] showed that in $\mathrm{Cu}$ and $\mathrm{Ni}$ new mechanisms of defect production occur when damage is close to a surface. In bcc materials, recent MD simulations in bcc Mo revealed the formation of large vacancy loops just below the surface [27] and Osetsky et al. have described the evolution of cascades close to the surface in bcc Fe [28].

In this work we focus on the differences between irradiation in Fe thin film and bulk material which has not been studied in detail up to now. In particular we focus on the formation of vacancy clusters, and their orientation, size and frequency. For this comparison we study the early stage of the damage produced by recoils of energies between $50 \mathrm{keV}$ and $100 \mathrm{keV}$ in thin films and bulk samples of bcc Fe at $0 \mathrm{~K}$ using MD simulations with 2 different empirical interatomic potentials. We assess and compare the number of vacancies and interstitials, and the size and morphology of their clusters immediately after the cool-down of the displacement cascade in the thin foil and bulk irradiation conditions, in order to identify the impact of the free surfaces. We intend to understand, in particular, whether in Fe thin foils vacancy loops of sizes visible in TEM could be formed directly in the cascade. We also discuss the various implications of extrapolating TEM in situ irradiation analysis to model radiation effects in bulk materials.

\section{Methodology}

The parallel MD code MDCASK developed at LLNL [29] was used for the calculations. Two different types of interatomic potentials were considered for comparison: one developed by Dudarev and Derlet [30] and one by Ackland et al. [31], cited respectively as DD and AM. Both potentials were modified for the high energyshort range interactions according to the procedure used in [32]. These potentials were also used previously to study cascade damage in bulk samples [33]. They were selected because they reproduce fairly well the stability of different point defects as obtained by density functional theory (DFT) calculations. The cluster size distribution obtained with these potentials also seems to reproduce experimental observations better than other potentials [33].

Simulations were performed in bcc Fe at constant volume. Two types of calculations were performed: one in a crystalline thin film oriented along a $\langle 001\rangle$ direction, and the other inside a crystal, quoted as 'bulk', with $\langle 001\rangle$ axes. The crystal thin foil is constructed with periodic boundary conditions along $x$ and $y$ axes, while both surfaces along the $z$ direction were free. An energetic Fe atom was launched from the outside of the crystal towards the surface with the selected implantation angle and energy. For the bulk crystal periodic boundary conditions were applied in all directions and one atom near the top of the simulation box was selected as the high-energy atom, or primary knock-on atom (PKA), and was given the selected angle and energy.

In order to minimize defect migration and to focus on the effect of surfaces on defect production, the temperature in all simulations was kept low, close to $0 \mathrm{~K}$. This was achieved by means of a thermal bath located at the border of the simulation box. The thermal bath consisted of two atomic layers where the velocity of the atoms was scaled every time step to correspond to the desired temperature of $0 \mathrm{~K}$. Calculations were followed for a period sufficient to reach a constant value for the number of defects produced. In this way simulated time amounts to about $20-40$ ps depending on the case. Between 14 and 30 simulations were performed for each condition studied in order to obtain statistically significant results. The incident angle, i.e., the angle of the incoming energetic ion with respect to a line perpendicular to the surface (which would correspond to the polar angle in a spherical coordinate system) is kept constant, while the azimuthal angle in that same coordinate system is varied via increasing it by $10^{\circ}$ for each different case. The initial random distribution of velocities of the atoms in the simulation box is also different for each case. Two energies, $50 \mathrm{keV}$ and $100 \mathrm{keV}$, were considered, and two incidence angles, $10^{\circ}$ and $22^{\circ}$, for each energy. We should mention that the critical angle for channeling according to the Lindhard expression [34] is $21^{\circ}$ for $50 \mathrm{keV}$ and $15^{\circ}$ for $100 \mathrm{keV}$ ions. The simulation conditions are summarized in Table 1 . The simulation volume for most conditions was $180 a_{0} \times 180 a_{0} \times 180 a_{0}$, where $a_{0}=2.8665 \AA$ is the lattice parameter for the Fe potentials considered here, which corresponds to a cube of about $50 \mathrm{~nm}$ a side. This size is comparable to the thickness of the thin films that are used experimentally in TEM in situ irradiations [12,13].

The analysis of the resulting damage was first conducted using the Wigner-Seitz cell method, which gives the number of vacancies and self-interstitials in the crystal. Secondly, once point defects were identified, their clusters were established using another method, which considers that two defects belong to the same cluster when the distance between them is between the first and second nearest neighbor of the bcc Fe lattice. Finally, the radius $R$ of a loop with $N$ defects was calculated according to the relationship [35] $R=a_{0} \sqrt{\frac{N}{2^{1 / 2} \pi}}$.

\section{Cascade damage: defect type and size}

We first present results for the thin foil geometry. Fig. 1 shows the damage microstructure of the Fe thin film 25 ps after launching the energetic ion for two different runs (with different azimuthal angles) under the same condition, i.e. $0 \mathrm{~K}$ with a $50 \mathrm{keV}$ Fe atom at an incidence angle of $10^{\circ}$ and with the AM potential. Green

\section{Table 1}

Irradiation conditions. The table shows the number of runs for each energy of the Fe ion in dependence of incidence angle, DD or AM interatomic potential, and thin film or bulk sample geometry used. The sample thickness is $51.4 \mathrm{~nm}$ except for $*$ and $* *$, where it is $45.7 \mathrm{~nm}$ and $70 \mathrm{~nm}$ respectively.

\begin{tabular}{|c|c|c|c|c|c|}
\hline \multirow[t]{2}{*}{ Energy (keV) } & \multirow[t]{2}{*}{ Incidence angle $\left({ }^{\circ}\right)$} & \multicolumn{2}{|c|}{$\begin{array}{l}\text { Thin foil } \\
\text { Number of } \\
\text { cascades } \\
\text { performed }\end{array}$} & \multicolumn{2}{|c|}{$\begin{array}{l}\text { Bulk } \\
\text { Number of } \\
\text { cascades } \\
\text { performed }\end{array}$} \\
\hline & & $\mathrm{DD}$ & $\mathrm{AM}$ & $\mathrm{DD}$ & $\mathrm{AM}$ \\
\hline 50 & $\begin{array}{l}10 \\
22\end{array}$ & $\begin{array}{r}14^{*} \\
17\end{array}$ & $\begin{array}{l}14 \\
30\end{array}$ & $\begin{array}{l}14 \\
14\end{array}$ & $\begin{array}{l}14 \\
14\end{array}$ \\
\hline 100 & $\begin{array}{l}10 \\
22\end{array}$ & $\begin{array}{l}20 \\
20\end{array}$ & $\begin{array}{l}20 \\
30\end{array}$ & - & - \\
\hline
\end{tabular}




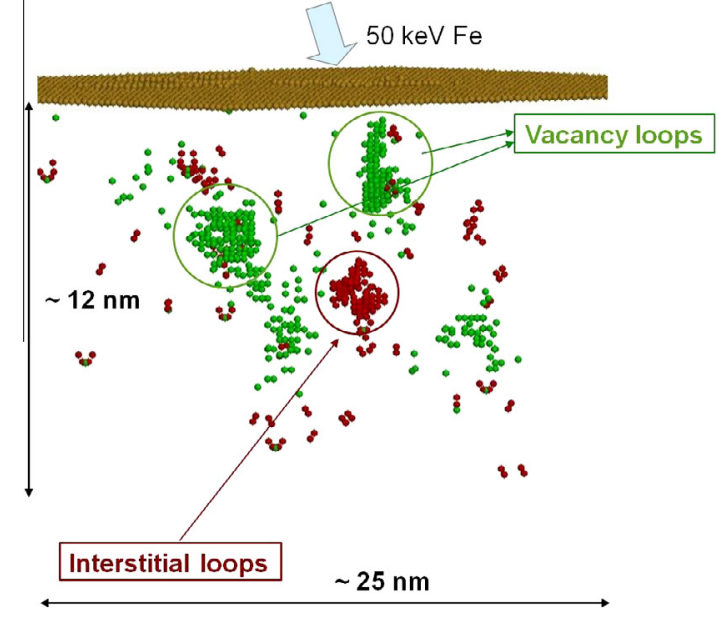

(a)

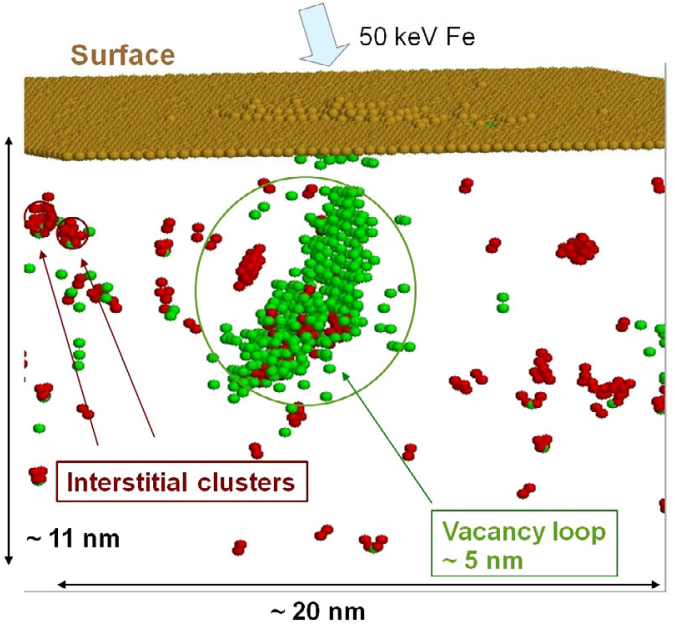

(b)

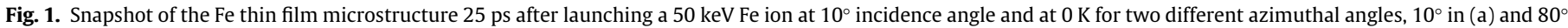

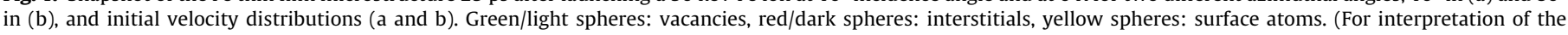
references to color in this figure legend, the reader is referred to the web version of this article.)

spheres mark the location of vacancies, while red spheres represent self-interstitials. The arrow indicates the approximate initial location of the energetic atom. Surface atoms are also represented in the figure with yellow spheres. In Fig. 1(a) the damage consists of isolated interstitials and vacancies, two neighboring relatively large clusters of 23 and 27 interstitials and two significantly larger clusters of 108 and 148 vacancies. The total number of vacancies in this case is 448 , while the total number of self-interstitials is 140 . $79 \%$ of the vacancies are in clusters and $57 \%$ of them are in clusters with more than 55 defects, which corresponds to a loop of about $1 \mathrm{~nm}$ in radius. $58 \%$ of the self-interstitials are in clusters but no cluster with more than 55 interstitials was found. As can be observed in the figure, most of the damage is located within $12 \mathrm{~nm}$ of the surface, while the displacement cascade reached a maximum depth of $20 \mathrm{~nm}$. Note that there are 308 ad-atoms at the free surface, as seen in Fig. 1(a). The number of missing selfinterstitial atoms corresponds to these ad-atoms.

Fig. 1(b) exhibits a clear difference to Fig. 1(a) even though the initial condition is the same, except for the azimuthal angle and the initial random distribution of velocities of the atoms in the simulation box. The difference in Fig. 1(b) consists of the absence of large interstitial clusters and the larger size of the vacancy loops; there is indeed one loop with 317 vacancies, much larger than any of those in Fig. 1(a). This is a trend we observed in the rest of the conditions: when a large vacancy loop is produced then only small interstitial clusters are obtained.

Fig. 2(a) shows the damage in an Fe thin film 20 ps after a displacement cascade induced by a $100 \mathrm{keV}$ Fe ion with a $10^{\circ}$ incidence angle, with the AM potential. The total number of resulting vacancies is 662 , while the total number of self-interstitials is 187 . As in Fig. 1, a significant number of atoms end up at the surface as ad-atoms (469), while 6 atoms are sputtered away. A large vacancy loop of 480 defects can be observed right below the surface. Fig. 2 (b) shows a closer view of this large cluster. Its dimensions are approximately $9 a_{0} \times 5 a_{0} \times 14 a_{0}$, or $3 \mathrm{~nm} \times 1 \mathrm{~nm} \times 4 \mathrm{~nm}^{3}$. It takes the form of a rectangular parallelepiped with a $(010)$ habit plane. This rectangular shape correlates well with the calculations by Gilbert et al. [36,37], showing that rectangular $\langle 100\rangle$ vacancy loops are energetically more stable than e.g. circular ones. Fig. 2 (c) shows a cross section of the specimen through the vacancy cluster, confirming the presence of a vacancy loop. The use of the RHFS rule gives a Burgers vector $\mathbf{b}=a_{0}[0-10]$ (Fig. 2(c)). Note that the presence of $\langle 100\rangle$ vacancy loops following displacement cascades in Fe was already shown by Soneda et al. [19] and Kapinos [38], but in bulk specimens. They also indicated that their formation was rare, constituting only $1 \%$ of all clusters.

We now present results for the bulk geometry. Fig. 3 shows the damage in bulk Fe 30 ps after a $50 \mathrm{keV}$ recoil with a $22^{\circ}$ angle of incidence, with the AM potential. The differences in the damage distribution and configuration between the bulk and thin foil conditions are clearly seen when compared with Fig. 1 . Note that as expected for the bulk the number of vacancies is the same as the number of self-interstitials, and equals 151 . A cluster with 38 vacancies and a relatively large cluster above it with 37 interstitials are observed.

Fig. 4 shows the damage in bulk Fe 22 ps after a $100 \mathrm{keV}$ recoil with an angle of $22^{\circ}$ and the AM potential for two different runs. There are differences: in the first case (Fig. 4(a)) small vacancy and self-interstitial clusters are observed; in the second case (Fig. 4(b)) slightly larger vacancy clusters are found, together with a few small self-interstitial clusters. We should note that, according to previous studies by Stoller and others [39], the breakdown into sub-cascades for Fe occurs at around $20 \mathrm{keV}$. Both cases here exhibit sub-cascade formation, visible with the different branches in the cascade.

\subsection{Statistical analysis}

As mentioned above, several authors have already reported some large vacancy loops following displacement cascades in the bulk $[19,33,38]$. Our simulations indicate that the frequency of formation of these large vacancy loops is greater when damage is produced in thin films, as seen in Figs. 1 and 2. In order to quantify this effect, we performed a statistical analysis of the data, focusing on the impact of the two different interatomic potentials. The number of vacancies and self-interstitials as well as their percentage in clusters were calculated for all conditions. Table 2 provides the mean values of the number of point defects, Table 3 lists their clustering fraction, and Table 4 provides the ion range projected along the [001] direction (i.e. the one perpendicular to the surface) for each condition. These values were obtained by fitting the different histograms to either a lognormal or a Gaussian distribution. 


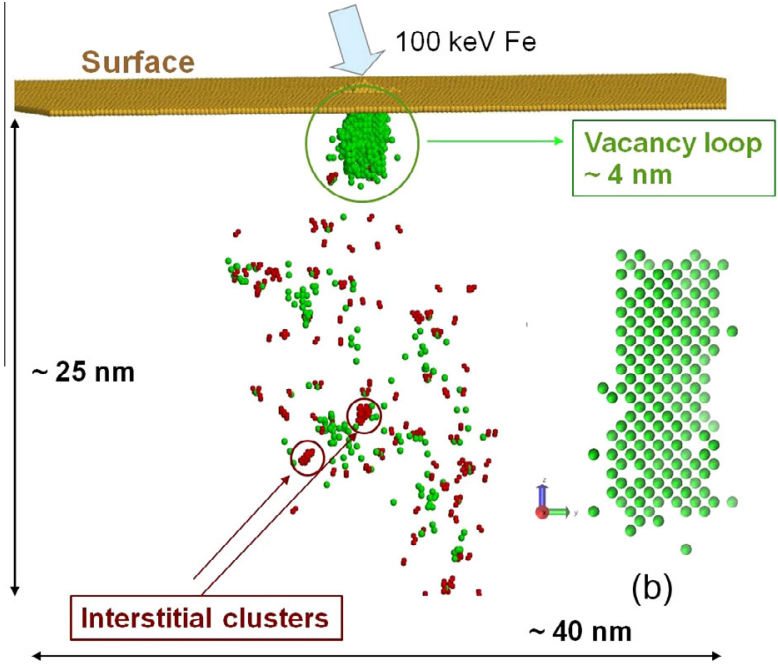

(a)

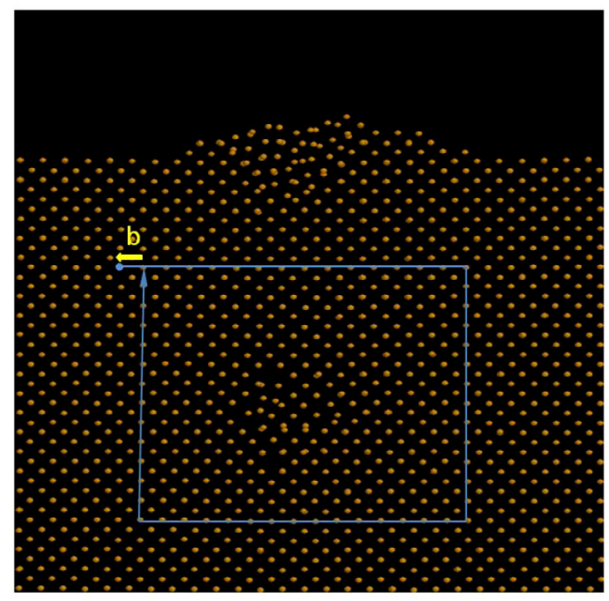

(c)

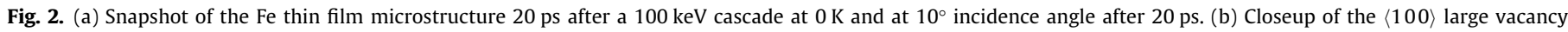

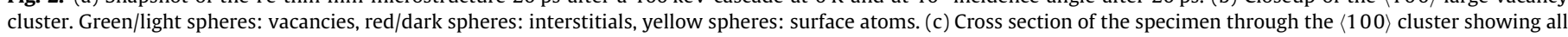

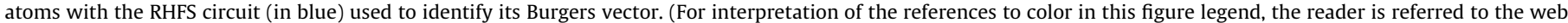
version of this article.)

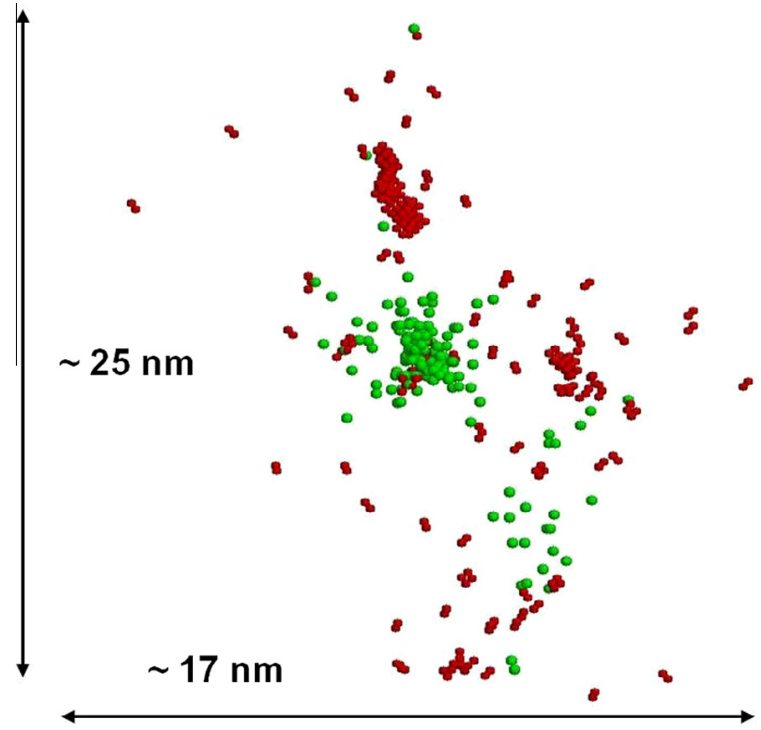

Fig. 3. Snapshot of the Fe bulk microstructure $30 \mathrm{ps}$ after a $50 \mathrm{keV}$ cascade at $0 \mathrm{~K}$ and at $22^{\circ}$ incidence angle. Green/light spheres: vacancies, red/dark spheres: interstitials. (For interpretation of the references to color in this figure legend, the reader is referred to the web version of this article.)

We first present the results for the thin film geometry. It can be seen in Table 2 that the number of self-interstitials is always lower than the number of vacancies. As already noted above, the missing self-interstitials correspond to atoms that have moved onto the surface, remaining as ad-atoms, as seen in Figs. 1 and 2. The number of atoms that are sputtered away is small. The maximum occurs for the $50 \mathrm{keV}$ Fe ion and $22^{\circ}$ incidence with the AM potential and constitutes $6 \%$ of the total number of interstitials. Table 2 shows that when the energy increases from $50 \mathrm{keV}$ to $100 \mathrm{keV}$ the damage exhibits an increase of between $50 \%$ and $200 \%$ for the number of vacancies and of between $10 \%$ and $110 \%$ for interstitials.

Fig. 5 presents the scatter in the data. It shows the histograms of the number of vacancies and self-interstitials obtained for the thin foil. Results for the $50 \mathrm{keV} \mathrm{Fe}$ ion with an incidence angle of $10^{\circ}$ are given in Fig. 5(a), and results for $100 \mathrm{keV}$ and $22^{\circ}$ incidence angle in Fig. 5(c). These were obtained with the AM potential. Fig. 5 (b) and (d) present the results for the DD potential for the $50 \mathrm{keV}$ Fe ion and $10^{\circ}$ incidence and the $100 \mathrm{keV}$ Fe ion and $22^{\circ}$ incidence, respectively. Several common features emerge from all of these cases.

It appears that the scatter in the number of defects from one run to another under the same irradiation condition is extensive. Some of the cases exhibit a significantly larger number of vacancies relative to the average of 300-400 vacancies. For example, for the condition of the $50 \mathrm{keV} \mathrm{Fe}$ ion, there is one case with close to 3000 vacancies (Fig. 5(a)), while for $100 \mathrm{keV}$ and the same potential (Fig. 5(c)) one case has more than 1000 vacancies. Fig. 6 shows the resulting damage configuration for one of these cases exhibiting a large number of vacancies: that with the AM potential, $50 \mathrm{keV}$ cascade and $10^{\circ}$ incidence. This damage is significantly different from that shown in Figs. 1-3. Even though the damage appears as a large vacancy cluster, it actually consists of a dislocated volume of the crystal with a crystalline structure in the center, which corresponds to a short dislocation array similar to that obtained by Ghaly and Averback [23]. We should point out that these cases are rare when using the AM potential but more frequent with the DD potential. For $50 \mathrm{keV}$ and $22^{\circ}$ incidence, $60 \%$ of the vacancy clusters with more than 20 vacancies correspond to surface damage in the case of the DD potential, whereas this is only $7 \%$ for the AM potential. As seen in Fig. 5(a)-(d), even if the extreme cases with a very large number of vacancies are not considered, the spread in the number of vacancies is still large, varying from 100 up to 600, i.e. there is still a variation of between $50 \%$ and $100 \%$.

We now present the results for the bulk geometry. Note that the slight differences between the mean value of vacancies and selfinterstitials (see Table 2) result from an inaccuracy in the method of calculating defects when large clusters are formed. In bulk cascades the number of defects produced does not seem to depend on the recoil direction. This applies to both interatomic potentials. In the case of the DD potential, the numbers of vacancies are 135 and 129 for recoil angles of $10^{\circ}$ and $22^{\circ}$, respectively. The values obtained for the AM potential are slightly higher: 159 and 164 


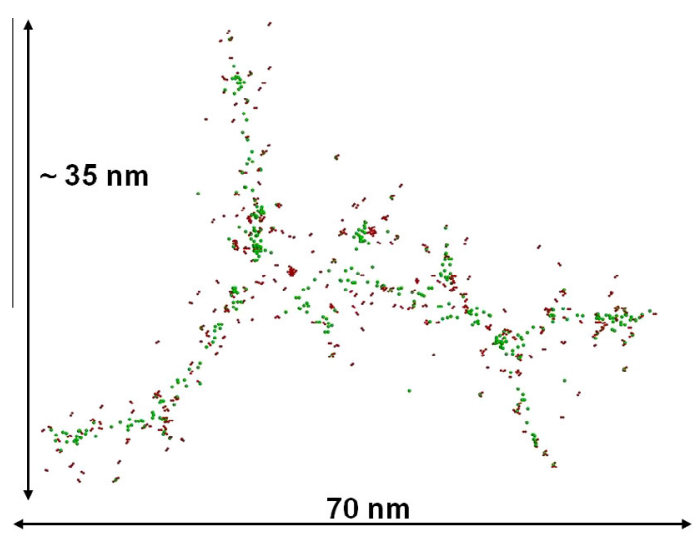

(a)

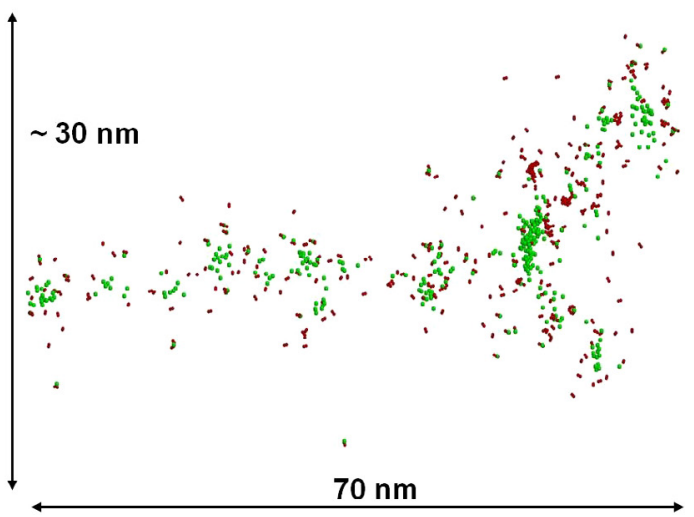

(b)

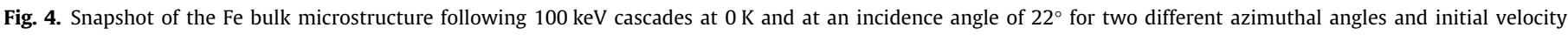

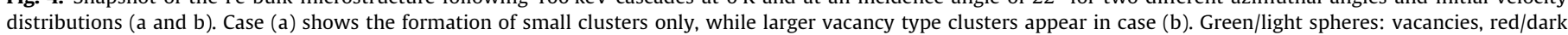
spheres: interstitials. (For interpretation of the references to color in this figure legend, the reader is referred to the web version of this article.)

Table 2

Average number of vacancies/interstitials following the displacement cascade in bcc Fe at $0 \mathrm{~K}$, depending on the irradiation and simulation condition.

\begin{tabular}{|c|c|c|c|c|c|}
\hline \multirow[t]{2}{*}{$\begin{array}{l}\text { Energy } \\
(\mathrm{keV})\end{array}$} & \multirow[t]{2}{*}{$\begin{array}{l}\text { Incidence } \\
\text { angle }\left(^{\circ}\right)\end{array}$} & \multicolumn{2}{|c|}{$\begin{array}{l}\text { Thin foil } \\
\text { (number of vacancies/ } \\
\text { interstitials) }\end{array}$} & \multicolumn{2}{|c|}{$\begin{array}{l}\text { Bulk } \\
\text { (Number of vacancies/ } \\
\text { interstitials) }\end{array}$} \\
\hline & & $\begin{array}{l}\text { DD } \\
\text { potential }\end{array}$ & $\begin{array}{l}\text { AM } \\
\text { potential }\end{array}$ & $\begin{array}{l}\text { DD } \\
\text { potential }\end{array}$ & $\begin{array}{l}\text { AM } \\
\text { potential }\end{array}$ \\
\hline \multirow[t]{2}{*}{50} & 10 & $173 / 135$ & $190 / 172$ & $135 / 125$ & $159 / 164$ \\
\hline & 22 & $130 / 125$ & $237 / 160$ & $129 / 122$ & $164 / 168$ \\
\hline \multirow[t]{2}{*}{100} & 10 & $257 / 148$ & $450 / 350$ & - & - \\
\hline & 22 & $380 / 267$ & $364 / 285$ & - & $333 / 334$ \\
\hline
\end{tabular}

the two potentials studied, with values between $38 \%$ and $45 \%$ of vacancies in clusters and between $32 \%$ and $53 \%$ for selfinterstitials in clusters. Note, however, that the fraction of interstitials in clusters for the DD potential is slightly larger than the fraction of vacancies in clusters. In the case of thin films, the fraction of vacancies in clusters is much larger than the fraction of self-interstitials in clusters in all cases. In addition, the increase in incident angle from $10^{\circ}$ to $22^{\circ}$ increases the clustering of vacancies by $13-30 \%$.

The examples of the damage produced in thin foils (Figs. 1 and 2) and in the bulk (Figs. 3 and 4) visually indicate that in thin films, compared to the bulk, larger vacancy clusters and smaller self-interstitial clusters are produced. Fig. 7 shows the cluster size distribution obtained for the $50 \mathrm{keV}$ cascades and $22^{\circ}$ incidence angle with the AM potential for thin films and bulk samples. Fig. 7(a) and (b) give the size distributions of clusters of vacancies and interstitials, respectively. They show clearly that thin film irradiation produces much larger vacancy clusters and slightly smaller interstitial ones than bulk material irradiation, with an average size of 140 vacancies and 30 interstitials for thin foil and 44 vacancies and 40 interstitials for the bulk. The clusters in the thin foil reach sizes that are visible in TEM.

Table 4 gives the mean value of the range of the initial energetic atom for the different conditions, excluding channeling cases. For the $50 \mathrm{keV}$ ion in the Fe thin film with an incidence angle of $10^{\circ}$ the mean value is $20 \mathrm{~nm}$ for the AM potential and $21 \mathrm{~nm}$ for the DD potential. Both have a standard deviation of $12 \mathrm{~nm}$. The value of the range obtained from the usual SRIM code [40] for this energy and angle is $18 \mathrm{~nm}$. Note that SRIM does not account for the crystalline structure of the target. For the $22^{\circ}$ impact angle and the DD potential the ion range increases by as much as $200 \%$ when their

Table 4

Ion range, in $\mathrm{nm}$, depending on the irradiation and simulation condition. High channeling cases are not included in the average.

\begin{tabular}{|c|c|c|c|c|c|}
\hline \multirow[t]{2}{*}{$\begin{array}{l}\text { Energy } \\
(\mathrm{keV})\end{array}$} & \multirow[t]{2}{*}{$\begin{array}{l}\text { Incidence } \\
\text { angle }\left(^{\circ}\right)\end{array}$} & \multicolumn{2}{|c|}{$\begin{array}{l}\text { Thin foil } \\
\text { Ion range in } \mathrm{nm}\end{array}$} & \multicolumn{2}{|c|}{$\begin{array}{l}\text { Bulk } \\
\text { Ion range in nm }\end{array}$} \\
\hline & & $\begin{array}{l}\text { DD } \\
\text { potential }\end{array}$ & $\begin{array}{l}\text { AM } \\
\text { potential }\end{array}$ & $\begin{array}{l}\text { DD } \\
\text { potential }\end{array}$ & $\begin{array}{l}\text { AM } \\
\text { potential }\end{array}$ \\
\hline \multirow[t]{2}{*}{50} & 10 & 21 & 20 & 10 & 11 \\
\hline & 22 & 7 & 11 & 12 & 8 \\
\hline \multirow[t]{2}{*}{100} & 10 & 22 & 26 & - & - \\
\hline & 22 & 21 & 27 & - & 32 \\
\hline
\end{tabular}

Table 3

Percentage of vacancies/interstitials in clusters following the displacement cascade in bcc Fe at $0 \mathrm{~K}$, depending on the irradiation and simulation condition.

\begin{tabular}{|c|c|c|c|c|c|}
\hline \multirow[t]{2}{*}{$\begin{array}{l}\text { Energy } \\
(\mathrm{keV})\end{array}$} & \multirow[t]{2}{*}{$\begin{array}{l}\text { Incidence } \\
\text { angle }\left(^{\circ}\right)\end{array}$} & \multicolumn{2}{|c|}{$\begin{array}{l}\text { Thin foil } \\
\text { (\% in clusters } \\
\text { vacancies/ } \\
\text { interstitials) }\end{array}$} & \multicolumn{2}{|c|}{$\begin{array}{l}\text { Bulk } \\
\text { (\% in clusters } \\
\text { vacancies/ } \\
\text { interstitials) }\end{array}$} \\
\hline & & $\begin{array}{l}\text { DD } \\
\text { potential }\end{array}$ & $\begin{array}{l}\text { AM } \\
\text { potential }\end{array}$ & $\begin{array}{l}\text { DD } \\
\text { potential }\end{array}$ & $\begin{array}{l}\text { AM } \\
\text { potential }\end{array}$ \\
\hline \multirow[t]{2}{*}{50} & 10 & $52 / 34$ & $55 / 38$ & $40 / 53$ & $39 / 32$ \\
\hline & 22 & $68 / 42$ & $62 / 38$ & $38 / 52$ & $45 / 43$ \\
\hline \multirow[t]{2}{*}{100} & 10 & $67 / 44$ & $42 / 37$ & - & - \\
\hline & 22 & $53 / 35$ & $54 / 38$ & - & 46/99 \\
\hline
\end{tabular}




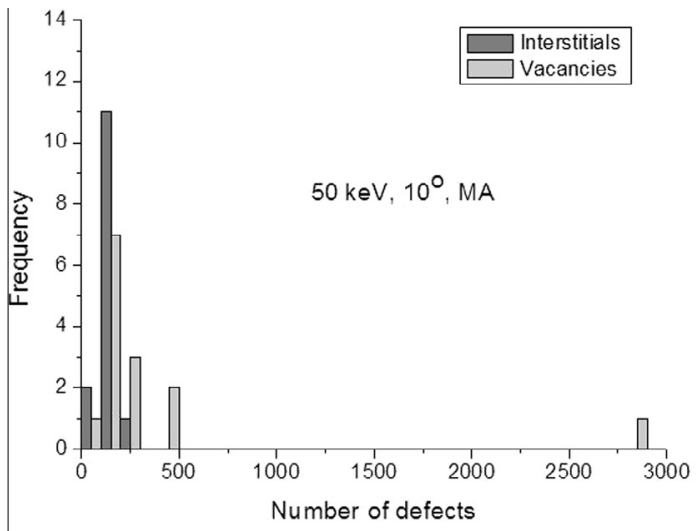

(a)

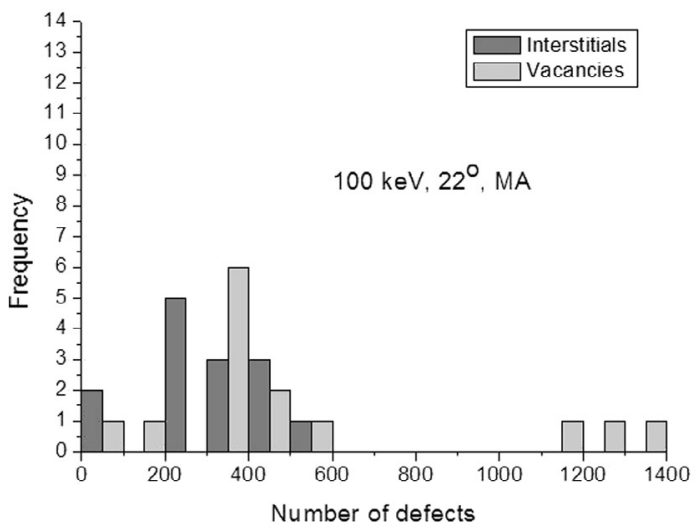

(c)

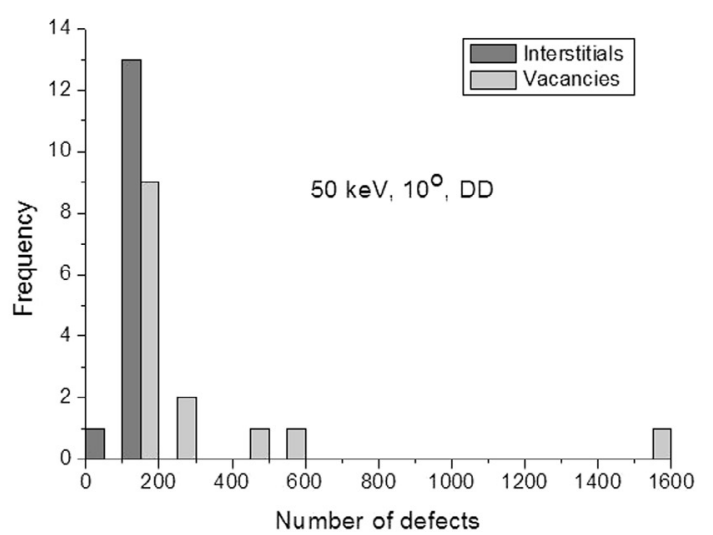

(b)

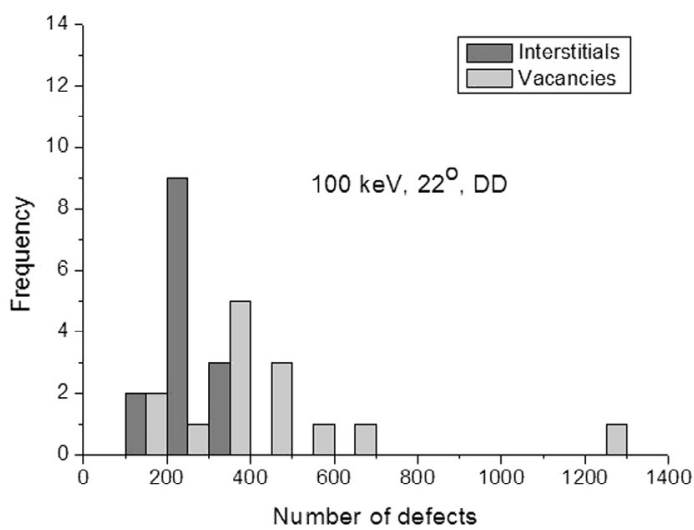

(d)

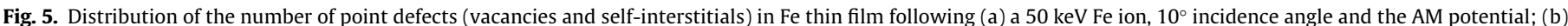

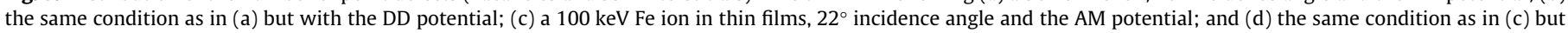
with the DD potential.
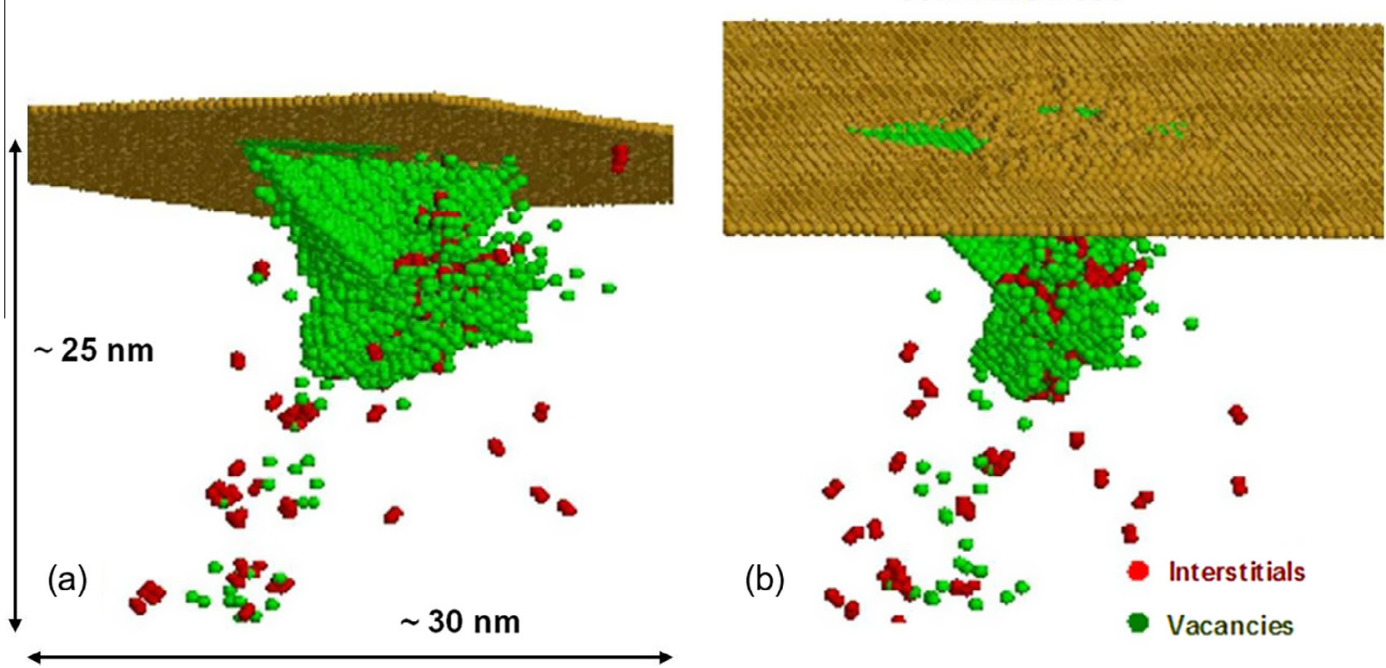

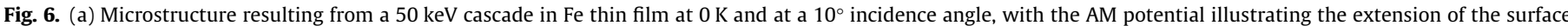

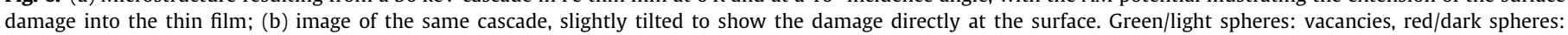
interstitials, yellow spheres: surface atoms. (For interpretation of the references to color in this figure legend, the reader is referred to the web version of this article.)

energy is increased from $50 \mathrm{keV}$ to $100 \mathrm{keV}$. As expected, the mean value of the ion range decreases when the incidence angle increases. Fig. 8 shows the distribution of the ion range obtained for the $50 \mathrm{keV}$ ion in the Fe thin film (Fig. 8(a) and (b)) and in the bulk (Fig. 8(c) and (d)), for all cases. Fig. 8(a) corresponds to an incidence angle of $10^{\circ}$ with both the AM potential and DD potential. As for the number of point defects, there is a wide spread in the values obtained, with some ions reaching only $5 \mathrm{~nm}$ while 
$50 \mathrm{keV}$, vacancies

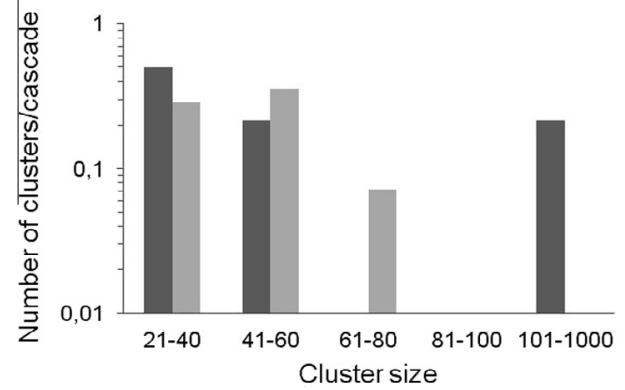

(a)
$50 \mathrm{keV}$, interstitials

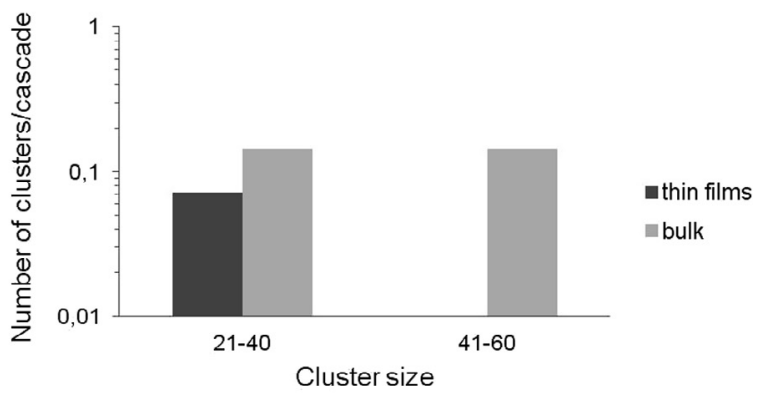

(b)

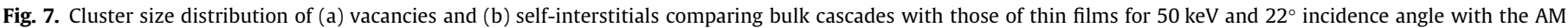
potential.

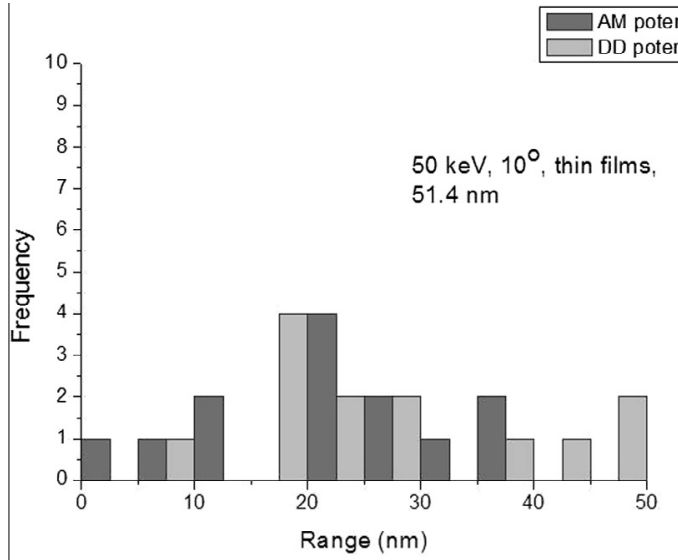

(a)

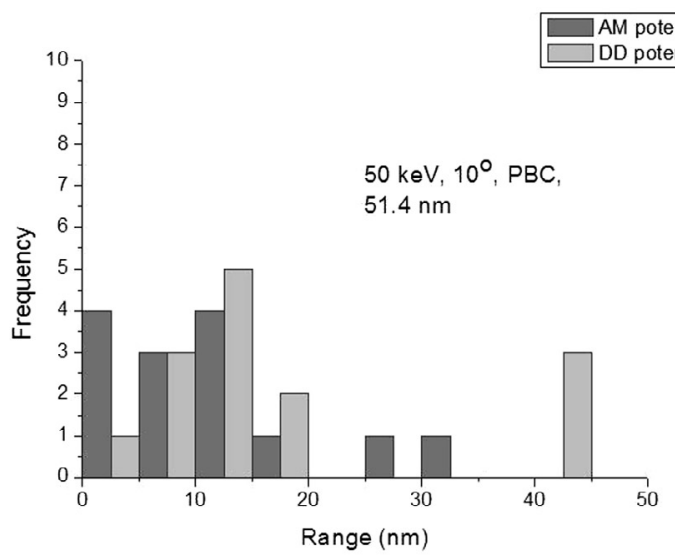

(c)

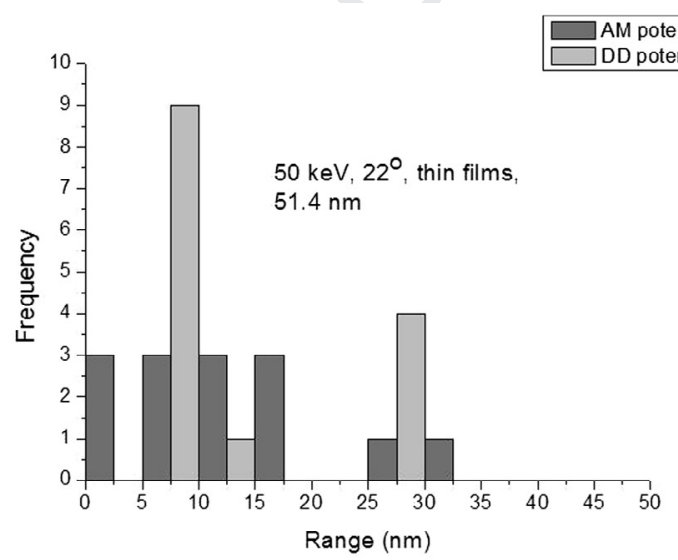

(b)

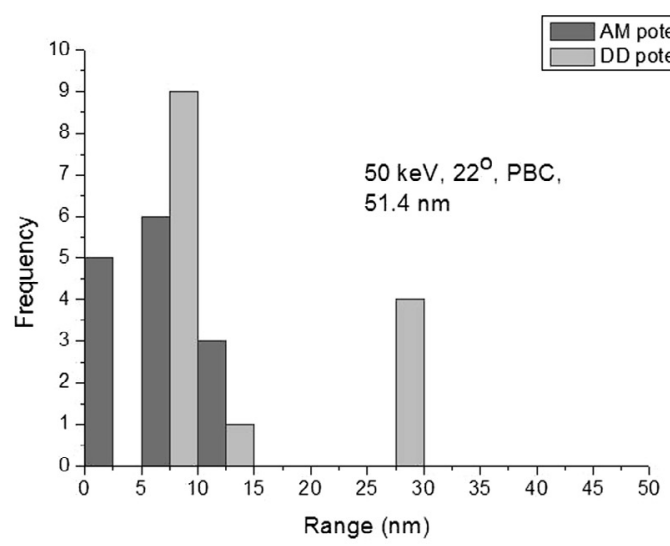

(d)

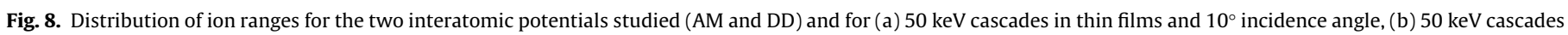
in thin films and $22^{\circ}$ incidence angle, (c) $50 \mathrm{keV}$ cascades in bulk and $10^{\circ}$ incidence angle, and (d) $50 \mathrm{keV}$ cascades in bulk and $22^{\circ}$ incidence angle.

others cross the whole sample thickness. In some cases the implanted ion is backscattered after colliding with the surface. Fig. 8(b), presenting the results for $50 \mathrm{keV}$ ions at $22^{\circ}$ incidence angle, again shows extensive scatter in the ion range, in particular for the AM potential. The ion range is on average $11 \mathrm{~nm}$ for the AM potential, with a standard deviation of $8 \mathrm{~nm}$, and $7 \mathrm{~nm}$ for the DD potential, with a standard deviation of $2 \mathrm{~nm}$.

Fig. 8(c) and (d) show the range distribution of the $50 \mathrm{keV} \mathrm{Fe}$ ion in bulk Fe for incidence angles of $10^{\circ}$ and $22^{\circ}$, respectively. The ion range distribution is narrower than for the thin film.
However, some channeling can be observed (particularly in the case of $10^{\circ}$ implantation), as expected from the Lindhard relationship [34], which leads to a greater ion range. As seen in Table 4, the mean values for the range obtained with the DD potential are similar for the two incidences studied: $10 \mathrm{~nm}$ with a standard deviation of $5 \mathrm{~nm}$ at $10^{\circ}$ incidence and $12 \mathrm{~nm}$ with a standard deviation of $8 \mathrm{~nm}$ at $22^{\circ}$ incidence. Similar values are obtained with the AM potential: $11 \mathrm{~nm}$ with a standard deviation of $9 \mathrm{~nm}$ at $10^{\circ}$ incidence and $8 \mathrm{~nm}$ with a standard deviation of $3 \mathrm{~nm}$ at $22^{\circ}$ incidence. 
in simulations. On the other hand, one should also keep in mind that for loops smaller than $1 \mathrm{~nm}$ the contrast in CTEM is reduced and the image size saturates (because of the diffraction-limited resolution), making the observation of loops more difficult, or impossible [45], which may also explain the lower yields observed in the experiment.

\section{Conclusions} the nature of the loops is cult to assess when they are smaller than $5 \mathrm{~nm}$. Jenkins et al. [14] showed the formation of $a_{0}\langle 100\rangle$ vacancy loops in Fe close to the surface after irradiation performed with $\mathrm{Ni}^{+}, \mathrm{Ge}^{+}, \mathrm{Kr}^{+}, \mathrm{Xe}^{+}$ and $\mathrm{W}^{+}$and energies between $40 \mathrm{keV}$ and $240 \mathrm{keV}$. More recently, Yao et al. [13] studied dislocation loops induced by $30 \mathrm{keV} \mathrm{Ga}^{+}$ions in $\mathrm{Fe}-11 \% \mathrm{Cr}$, which produced damage within $10 \mathrm{~nm}$ from the surface. They were able to determine that the loops (at least those closest to the surface) are of vacancy type.

For comparison with this experimental evidence we simulated a $30 \mathrm{keV} \mathrm{Ga}^{+}$ion implanted in an $\alpha$-Fe matrix. The AM interatomic potential was used for $\mathrm{Fe}-\mathrm{Fe}$ interactions, while the interaction between the $\mathrm{Ga}^{+}$ion and the Fe atoms was calculated using a pure repulsive potential, the so-called Universal potential described in [43]. In this way the damage produced by this ion as it travels through the lattice is described well. Fig. 9 shows the defects produced by the $30 \mathrm{keV}$ Ga ion after $17 \mathrm{ps}$. The total number of vacancies in this case is 799 and the total number of interstitials is 107. A large ad-atom island at the surface with 627 ad-atoms is seen. The formation of a large $\langle 100\rangle$ vacancy loop close to the surface is also clearly observed. It has 692 vacancies and is approximately 15 $a_{0} \times 9 a_{0} \times 21 a_{0}$, or $4 \mathrm{~nm} \times 3 \mathrm{~nm} \times 6 \mathrm{~nm}$. This cluster is comparable to those observed experimentally by Yao et al. [13], who performed these experiments with $\mathrm{Fe}-11 \% \mathrm{Cr}$. As shown, however, in MD calculations by Malerba et al. [44] the damage produced in the cascade in $\alpha$-Fe and $\mathrm{FeCr}$ alloys is not significantly different. The only difference is in the self-interstitial loops, which in $\mathrm{FeCr}$ alloys can be a mixture of $\mathrm{Fe}$ and $\mathrm{Cr}$ atoms.

We should point out that experiments by Robertson et al. $[41,42]$ using low-energy Fe ions (50 keV and $100 \mathrm{keV}$ ) in Fe show yields for the formation of loops much lower than those found in the simulations presented here. A probable cause for this discrepancy is the difference in time scales between simulations and experiments. The simulations have been performed for tens of picoseconds. For longer time scales, it is, however, conceivable that vacancy loops close to the surface are able to climb and disappear by recombination, while self-interstitial clusters may coalesce and form larger loops, resulting in a yield lower than the one obtained
The calculations presented here show that the damage produced by ion implantation in Fe thin film using $50 \mathrm{keV}$ and $100 \mathrm{keV}$ ions is significantly different from that produced in bulk Fe by recoils of the same energy. In thin films, results show the formation of $\langle 100\rangle$ vacancy loops with sizes visible in the TEM. This results from the imbalance in the number of vacancies with respect to self-interstitials, due to the trapping of the latter at the surface where they remain as ad-atoms.

Statistical analysis reveals a large dispersion in the defects produced: while bulk results present a narrow dispersion in terms of the total number of defects or the percentage of defects in clusters, in thin foil the total number of defects varies significantly from one cascade to another, as does the morphology of the damage produced. Two types of structures were identified in the thin foil. On the one hand, there are those exhibiting small self-interstitial clusters and large vacancy clusters right below the surface in the form of large $\langle 100\rangle$. On the other hand, there are structures presenting an array of dislocations and ad-atoms. The latter are, however, rare. Both the AM and DD potentials induce equivalent results in terms of damage, although morphologically the DD potential produces more frequent surface damage of the type shown in Fig. 6.

A larger fraction of vacancies in clusters are found in thin films than in the bulk, independently of the energy, angle of incidence or interatomic potential used. These vacancy clusters are also larger. The inverse behavior is observed for self-interstitials: in most cases, the fraction of self-interstitials in clusters is higher in the bulk and their sizes are larger than in the thin film. Increasing the energy from $50 \mathrm{keV}$ to $100 \mathrm{keV}$ shows the formation of sub-cascades in the case of bulk irradiation, resulting in smaller self-interstitial clusters for the higher energy. Sub-cascade formation at $100 \mathrm{keV}$ in the thin film can be seen only in some cases.

The formation of large $\langle 100\rangle$ vacancy loops directly in the casHowever, the nature of these loops has been experimentally identified only in the case of irradiation of $\mathrm{Fe}-11 \% \mathrm{Cr}$ with $\mathrm{Ga}^{+}$ions of lower energy (30 keV) [13], or irradiation of Fe with heavy ions cade revealed here for Fe thin film agrees well with experiments.

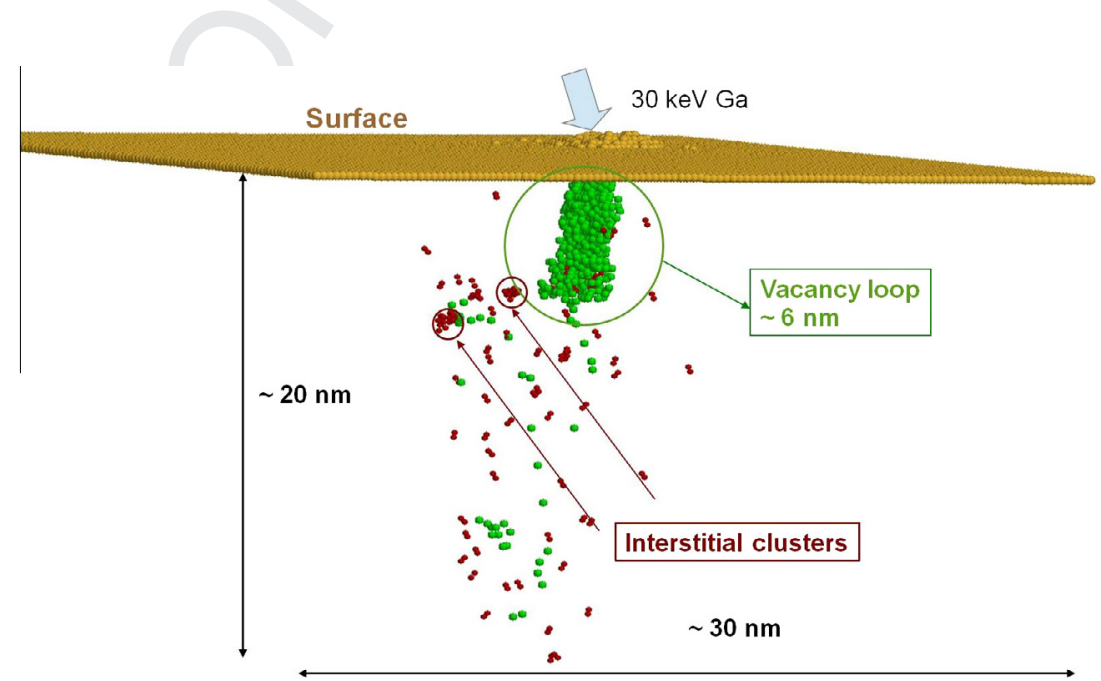

Fig. 9. Microstructure resulting from a cascade in an Fe thin film at $0 \mathrm{~K}$ induced by a $30 \mathrm{keV} \mathrm{Ga} \mathrm{ion} \mathrm{at} 22^{\circ}$ incidence angle. 
(40-240 keV) [16]. Our simulations show that these loops also form when irradiating with Fe ions of $50 \mathrm{keV}$ and $100 \mathrm{keV}$. These loops are, however, smaller than those produced in the Ga irradiation experiment at lower energy, and are therefore presumably more difficult to observe and analyze experimentally.

In previous works $[33,35]$ it is shown that the initial damage in the cascade together with defect mobilities define how damage will grow with dose. This has consequences for the modeling of irradiation effects. In the quest to develop models that are able to describe neutron damage in the bulk, ion implantation experiments using thin films are often used for validation. Our results show that one should carefully account for the effect of free surfaces in these models.

\section{Acknowledgments}

We would like to thank Drs. A. Prokhodtseva, M. HernándezMayoral, Z. Yao and S. Dudarev for fruitful discussions. Simulations were carried out using the computer cluster of the Dept. of Applied Physics at the UA, the HPC-FF supercomputer of the Jülich Supercomputer Center (Germany) and the Helios supercomputer at Rokkasho (Japan). MJA thanks the UA for support through an institutional fellowship. The research leading to these results is partly funded by the European Atomic Energy Community's (Euratom) Seventh Framework Programme FP7/2007-2013 under Grant agreement No. 604862 (MatISSE project) and in the framework of the EERA (European Energy Research Alliance) Joint Programme on Nuclear Materials and the Generalitat Valenciana PROMETEO2012/011. This work has been carried out within the framework of the EUROfusion Consortium and has received funding from the Euratom research and training programme 2014-2018 under Grant agreement No. 633053. The views and opinions expressed herein do not necessarily reflect those of the European Commission.

\section{References}

[1] J.L. Boutard, A. Alamo, R. Lindau, M. Rieth, Fissile core and tritium-breeding blanket: structural materials and their requirements, C. R. Phys. 9 (2008) 287

[2] S.J. Zinkle, G.E. Ice, M.K. Miller, S.J. Pennycook, Z.-L. Wang, Advances in microstructural characterization, J. Nucl. Mater. 386-388 (2009) 8-14.

[3] <http://jannus.in2p3.fr/>.

[4] C.W. Allen, L.L. Funk, E.A. Ryan, New instrumentation in Argonne's HVEMtandem facility: expanded capability for in situ ion beam studies, in: Mat. Res. Soc. Symp. Proc., vol. 396, 1996, p. 641.

[5] Y. Matsukawa, S.J. Zinkle, One-dimensional fast migration of vacancy clusters in metals, Science 318 (2007) 959.

[6] B.C. Masters, Dislocation loops in irradiated iron, Nature 200 (1963) 254

[7] W.M. Stobbs, C.H. Sworn, The weak beam technique as applied to the determination of the stacking-fault energy of copper, Philos. Mag. 24 (1971) 1365.

[8] R. Schaublin, J. Henry, Y. Dai, Helium and point defect accumulation: (i) Microstructure and mechanical behaviour, C. R. Phys. 9 (2008) 389-400.

[9] K. Arakawa, K. Ono, M. Isshiki, K. Mimura, M. Uchikoshi, H. Mori, Observation of the one-dimensional diffusion of nanometer-sized dislocation loops, Science 318 (2007) 956

[10] N. Soneda, T. Diaz de la Rubia, Defect production, annealing kinetics and damage evolution in alpha-Fe: an atomic-scale computer simulation, Philos. Mag. A 78 (1998) 995.

[11] Yu.N. Osetsky, D.J. Bacon, A. Serra, B.N. Singh, S.I. Golubov, Stability and mobility of defect clusters and dislocation loops in metals, J. Nucl. Mater. 276 (2000) 65.

[12] A. Prokhodtseva, B. Décamps, R. Schäublin, Comparison between bulk and thin foil ion irradiation of ultra high purity Fe, J. Nucl. Mater. 442 (2013) S786S789.

[13] Z. Yao, M. Hernández Mayoral, M.L. Jenkins, M.A. Kirk, Heavy-ion irradiations of $\mathrm{Fe}$ and $\mathrm{Fe}-\mathrm{Cr}$ model alloys Part 1: Damage evolution in thin-foils at lower doses, Philos. Mag. 88 (2008) 2851.

[14] M.L. Jenkins, Z. Yao, M. Hernández-Mayoral, M.A. Kirk, Dynamic observations of heavy-ion damage in Fe and Fe-Cr alloys, J. Nucl. Mater. 389 (2009) 197202.
[15] M. Hernández-Mayoral, Z. Yao, M.L. Jenkins, M.A. Kirk, Heavy-ion irradiations of $\mathrm{Fe}$ and $\mathrm{Fe}-\mathrm{Cr}$ model alloys Part 2: Damage evolution in thin-foils at higher doses, Philos. Mag. 88 (2008) 2881.

[16] M.L. Jenkins, C.A. English, B.L. Eyre, Heavy-ion damage in alpha Fe, Nature 263 (1976) 400-401.

[17] D.J. Bacon, A.F. Calder, F. Gao, Defect production due to displacement cascades in metals as revealed by computer, J. Nucl. Mater. 251 (1997) 1.

[18] R.E. Stoller, G.R. Odette, B.D. Wirth, Primary damage formation in bcc iron, J. Nucl. Mater. 251 (1997) 49-60.

[19] N. Soneda, S. Ishino, T.D. de la Rubia, Vacancy loop formation by 'cascade collapse' in alpha-Fe: a molecular dynamics study of $50 \mathrm{keV}$ cascades, Philos. Mag. Lett. 81 (2001) 649.

[20] E. Zarkadoula, S.L. Daraszewicz, D.M. Duffy, M.A. Seaton, I.T. Todorov, K. Nordlund, M.T. Dove, K. Trachenko, The nature of high-energy radiation damage in iron, J. Phys.: Condens. Matter 25 (2013) 125402.

[21] L. Malerba, Molecular dynamics simulation of displacement cascades in alphaFe: a critical review, J. Nucl. Mater. 351 (2006) 28-38.

[22] A.F. Calder, D.J. Bacon, A.V. Barashev, Yu.N. Osetsky, On the origin of large interstitial clusters in displacement cascades, Philos. Mag. 90 (2010) 863.

[23] A.E. Sand, S.L. Dudarev, K. Nordlund, High-energy collision cascades in tungsten: dislocation loops structure and clustering scaling laws, Eur. Phys. Lett. 103 (2013) 46003

[24] M. Ghaly, R.S. Averback, Effect of viscous-flow on ion damage near solidsurfaces, Phys. Rev. Lett. 72 (1994) 364.

[25] K. Nordlund, J. Keinonen, M. Ghaly, R.S. Averback, Coherent displacement of atoms during ion irradiation, Nature 398 (1999) 49.

[26] K. Nordlund, J. Keinonen, M. Ghaly, R.S. Averback, Recoils, flows and explosions: surface damage mechanisms in metals and semiconductors during $50 \mathrm{eV}-50 \mathrm{keV}$ ion bombardment, Nucl. Instrum. Methods Phys. Res. B 148 (1999) 74-82.

[27] S.V. Starikov, Z. Insepov, J. Rest, A.Y. Kuksin, G.E. Norman, V.V. Stegailov, A.V. Yanikin, Radiation-induced damage and evolution of defects in Mo, Phys. Rev. B 84 (2011) 104109.

[28] Yu.N. Osetsky, A.F. Calder, R.E. Stoller, How do energetic ions damage metallic surfaces? Curr. Opin. Solid State Mater. Sci., in press.

[29] T. Diaz de la Rubia, M.W. Guinan, New mechanism of defect production in metals: a molecular-dynamics study of interstitial-dislocation-loop formation in high-energy displacement cascades, Phys. Rev. Lett. 66 (1991) 2766.

[30] S. Dudarev, P. Derlet, A 'magnetic' interatomic potential for molecular dynamics simulations, J. Phys: Condens. Matter 17 (2005) 1-22.

[31] G.J. Ackland, M.I. Mendelev, D.J. Srolovitz, S. Han, A.V. Barashev, Development of an interatomic potential for phosphorus impurities in alpha-iron, J. Phys: Condens. Matter 16 (2004) S2629.

[32] C. Björkas, K. Nordlund, Comparative study of cascade damage in Fe simulated with recent potentials, Nucl. Instrum. Methods Phys. Res. B 259 (2007) 853860.

[33] C. Björkas, K. Nordlund, M.J. Caturla, Influence of the picosecond defect distribution on damage accumulation in irradiated alpha-Fe, Phys. Rev. B 85 (2012) 024105

[34] L.-P. Zheng, Z.Y. Zhu, Y. Li, F.O. Goodman, An improved critical angle equation for channeling, Nucl. Instrum. Methods Phys. Res. B 268 (2010) 120-122.

[35] M.J. Caturla, N. Soneda, E. Alonso, B.D. Wirth, T.D. de la Rubia, J.M. Perlado, Comparative study of radiation damage accumulation in $\mathrm{Cu}$ and $\mathrm{Fe}$, J. Nucl. Mater. 276 (2000) 13.

[36] M.R. Gilbert, S.L. Dudarev, P.M. Derlet, D.G. Pettifor, Structure and metastability of mesoscopic vacancy and interstitial loop defects in iron and tungsten, J. Phys.: Condens. Matter 20 (2008) 345214.

[37] M.R. Gilbert, Z. Yao, M.A. Kirk, M.L. Jenkins, S.L. Dudarev, Vacancy defects in Fe: comparison between simulation and experiment, J. Nucl. Mater. 386-388 (2009) 36-40.

[38] V.G. Kapinos, Y.N. Osetskii, P.A. Platonov, The mechanism of nucleation of vacancy loops with Burgers vectors (100) in bcc metals, J. Nucl. Mater. 173 (1990) 229.

[39] R.E. Stoller, in: R.J.M. Konings (Ed.), Comprehensive Nuclear Materials, vol. 1, Elsevier, Amsterdam, 2012, p. 293.

[40] J.F. Ziegler, J.P. Biersack, The stopping and range of ions in matter, SRIM-2003, Ó1998, 1999 by IB77M co.

[41] I.M. Robertson, M.A. Kirk, W.E. King, Formation of dislocation loops in iron by self-ion irradiations at $40 \mathrm{~K}$, Scr. Met. 18 (1984) 317.

[42] M.A. Kirk, I.M. Robertson, M.L. Jenkins, C.A. English, T.J. Black, J.S. Vetrano, The collapse of defect cascades to dislocation loops, J. Nucl. Mater. 149 (1987) 21; Kirk, et al., ASTM Spec. Tech. Pub. 955 (1987) 48.

[43] J.P. Biersack, J.F. Ziegler, Refined universal potentials in atomic-collisions, Nucl. Instrum. Methods 194 (1982) 93.

[44] L. Malerba, D. Terentyev, P. Olsson, R. Chakarova, J. Wallenius, Molecular dynamics simulation of displacement cascades in $\mathrm{Fe}-\mathrm{Cr}$ alloys, J. Nucl. Mater. 329-333 (2004) 1156.

[45] R. Schäublin, A. Almazouzi, Y. Dai, Yu.N. Osetsky, M. Victoria, Quantitative analysis of CTEM images of small dislocation loops in $\mathrm{Al}$ and stacking fault tetrahedral in $\mathrm{Cu}$ generated by molecular dynamics simulation, J. Nucl. Mater. 276 (2000) 251. 